\title{
Influencia del clima sociofamiliar y estilos de interacción parental sobre el desarrollo de habilidades sociales en niños y niñas
}

\author{
Laura Isaza Valencia \\ Universidad Pontificia Bolivariana, Medellín \\ Gloria Cecilia Henao López \\ Universidad Eafit, Medellín
}

Recibido: 12 de enero del 2012 / Aprobado: 21 de febrero del 2012

Este estudio descriptivo explicativo analiza la influencia de los estilos de interacción parental y del clima social familiar sobre el desarrollo de habilidades sociales de 108 niños y niñas. Los instrumentos utilizados fueron la escala de prácticas educativas familiares de García y Román (2003), la escala de clima social de Moos y Ticket (1974) y el inventario de Monjas (2000). Los resultados muestran que a mayor presencia de acciones equilibradas y mayor clima de participación, los niños y niñas presentan un mayor desempeño social, y a mayor utilización de estrategias autoritarias hay un menor desarrollo de repertorios sociales.

contexto familiar / clima social / estilos de interacción / desempeño social

Influence of socio-familiar climate and parent interaction styles on the development of social abilities on children

This descriptive and explanatory study analyzed the influence of parenting styles interaction and social family climate on the development of social skills of 108 children. Data where collected by these instruments: Family Educational Practices Scale of Roman Garcia (2003), Social Climate Scale of Moos and Ticket (1974), and Nuns Inventory (2000). Results showed than the higher presence of balanced actions and participative climate, the better social performance children showed. Furthermore, the higher use of authoritarian strategies will diminish the development of social repertories.

family environment / social climate / interacting styles / social performance

Correos electrónicos: isaza8888@gmail.com,gloriach@une.net.co 


\section{INTRODUCCIÓN}

En el estudio del desarrollo infantil, juega un papel esencial la familia, principalmente las acciones educadoras de padres, madres y adultos significativos y su forma de relacionarse con sus hijos e hijas. El reconocimiento de este rol de la familia ha llevado a estudiar las actitudes, creencias, valores y acciones de crianza y formación de padres, madres y cuidadores inmediatos, que se manifiestan en las interacciones dinámicas y recíprocas presentes entre estos y el niño en la cotidianidad del hogar. Desde esta consideración de la familia, resulta importante conocer las acciones de crianza presentes en los padres y las madres y su relación con el desarrollo de las dimensiones social, emocional, cognitiva, comunicativa, moral y corporal, para evaluar apropiadamente el ejercicio de la paternidad, apoyar un adecuado ejercicio de la crianza y corregir las dificultades presentes entre padres, madres, hijos e hijas (Bornstein et al., 2011, Lansford et al., 2011, Lansford \& Bornstein, 2011).

La familia como contexto educativo ha sido estudiada como sistema familiar de socialización de los niños y las niñas desde los presupuestos ecológico, contextualista y socialculturalista. La mirada ecológica de Bronfenbrenner (1987) resalta la importancia del contexto familiar como sistema social y explica la relación existente entre las influencias ambientales y el desarrollo; estudió el desarrollo desde la observación del niño y la niña cuando interactúan con los padres y las personas cercanas en sus espacios cotidianos. La mirada contextualista exalta la experiencia social del niño y la niña para construir los aprendizajes, los padres se conciben como la fuente socializadora de sus hijos e hijas, quienes con sus acciones y verbalizaciones posibilitan una adquisición de repertorios sociales, comunicativos, emocionales y cognitivos (Sroufe, 2000, Eisenberg, Valiente, Morris, Fabes, Cumberland \& Reiser, 2003). La perspectiva socialculturalista enfatiza que la familia impulsa a los niños y niñas a progresar en el aprendizaje, y los padres, mediante las interacciones que establecen con sus hijos e hijas, posibilitan aprendizajes de repertorios y alcances de competencias en las diversas dimensiones (cognitiva, comunicativa, socioafectiva, corporal, estética, espiritual y ética). Los padres y madres ejercen una participación guiada en el proceso de adquisición de aprendizaje de los hijos e hijas, permitiendo que estos se dirijan de una zona de desarrollo real a una zona de desarrollo proximal; que se hace evidente cuando el niño o la niña logran en un primer momento hacer una actividad en compañía del adulto, para conseguir posteriormente desplegar la capacidad de solucionar independientemente esa actividad (Vigotsky, 1979; Rogoff, 1993).

La acción parental se concibe como una condición fundamental para el desarrollo infantil, debido a que las accio- 
nes y los hábitos cotidianos manifestados en las respuestas que los padres y las madres brindan a las demandas de sus hijos e hijas, las formas y manifestaciones de afecto, las acciones disciplinarias y las particularidades psicosociales e institucionales de la familia generan un clima alfabetizador y educativo protector o limitante del desarrollo; estas acciones y hábitos se manifiestan en pautas, estrategias o estilos de interacción particulares (autoritario, equilibrado y permisivo) y en una clima y estructura dinámica cohesiva, disciplinada o no orientada.

Los estilos de interacción familiar y el clima social familiar presentes en padres, madres y adultos juegan un papel fundamental en el aprendizaje de habilidades y repertorios sociales de sus hijos e hijas, en la construcción de competencias comunicativas, en el despliegue del desempeño emocional y en el desarrollo de bases alfabetizadoras.

\section{Estilos de interacción familiar}

Los estilos de interacción familiar son asumidos como procesos interactivos de naturaleza bidireccional que ocurren de manera continua y compleja, que se refieren a comportamientos de los padres y madres manifestados para guiar a los niños y niñas hacia el alcance de los fines de la socialización. Son portadores de significaciones sociales, que se sitúan en el contexto de las interacciones familiares. En suma, los estilos de interacción son un conjunto de acciones y repertorios conductuales aprendidos por los padres y madres que buscan guiar y conducir las conductas de los niños y las niñas, que se fundan en la propia educación vivida y en la imitación de sus padres y otros referentes cercanos (Aguirre, 2000; Solis-Cámara \& Díaz, 2007).

Dependiendo de las manifestaciones de afecto, los espacios y las formas de comunicación, así como las estrategias de control utilizadas por los padres y las madres para la conducción y contención de la conducta de sus hijos e hijas, se determinan los estilos de interacción presentes: autoritario (la estrategia utilizada por los padres es impositiva y arbitraria, utilizando la intimidación verbal, física y de privaciones); equilibrado (padres que dan importancia a lo que piensa el niño o la niña, expresan apoyo y afecto, presentan control y propician de manera efectiva la comunicación), o permisivo (padres con un control parental flexible, limitado y ausente, generan una comunicación unidireccional y poco efectiva). Estas manifestaciones dependen de la lectura que los padres y las madres tienen de sus hijos e hijas, de la madurez que esperan estos desarrollen, de sus propias estructuras de personalidad, de la crianza experimentada con sus padres y de las características socioculturales. Dimensiones como el control, el afecto y el grado de implicación en la crianza de los hijos y las hijas forman bases esenciales, convir- 
tiéndose en componentes que sustentan la presencia de un estilo educativo y las particularidades de las relaciones entre los miembros de la familia (Grusec et al., 2000; Mestre et al., 2001, 2003).

Los estilos de interacción han sido también nombrados como estilos de crianza (Baumrind, 1970, 1971); prácticas educativas familiares (García \& Román, 2003) o prácticas de crianza (Aguirre, 2000; Solís-Cámara \& Díaz 2007), y se han relacionado con diversas dimensiones psicológicas (autoconcepto, aptitudes, inteligencia, motivación, desarrollo emocional y social), sociodemográficas (familia, sexo, clase social, estructura familiar, nivel educativo de los padres) y escolares (métodos de enseñanza, ambiente escolar, rendimiento académico, estilos de aprendizaje). Los estudios de estos estilos se inician con los trabajos de Baumrind (1970, 1971), que se enfocaron en la relación existente entre la conducta de los padres y la estructuración de la personalidad de sus hijos e hijas; esta autora se centró en el estilo autoritario, autoritativo, permisivo y negligente. En sus estudios se afirma que el estilo de relación parental se asocia con la adquisición de características socioemocionales, en las cuales los niños y las niñas presentan confianza en sí mismos cuando los padres usan estrategias autoritativas; por el contrario, los hijos y las hijas de padres permisivos, negligentes y autoritarios se muestran ansiosos, inquietos y deprimidos.
Entre los estudios que parten del modelo teórico de Baumrind se encuentra el realizado por García, Ramírez y Lima (1998), quienes estudiaron la relación presente entre las prácticas educativas familiares y la construcción de valores en familia. En este estudio se encontró que los estilos educativos parentales se relacionan de manera positiva o negativa con el aprendizaje de valores prosociales de los niños y las niñas, en la autodirección y en el logro de aprendizajes significativos. Estos autores encontraron que aquellos niños con padres autoritarios asumen y aceptan las demandas de manera sumisa. Los padres permisivos generan una comunicación unidireccional y poco efectiva. Los padres democráticos generan aprendizajes significativos en los niños y las niñas.

Otros estudios coinciden en resaltar la asociación existente entre los estilos de paternidad y el desarrollo infantil, los padres presentan un rol fundamental en el desarrollo cognitivo, social y emocional de sus hijos e hijas, siendo los padres permisivos retardadores del desarrollo, los padres autoritarios limitantes del desempeño y los padres democráticos formadores de niños y niñas seguros, independientes, adaptados socialmente y exitosos. De esta manera, los estilos de interacción familiar se relacionan con la empatía y el autoconcepto, pero también con la agresividad, la inestabilidad emocional y la ira. (Fernández, 2003; García \& Román, 
2005; Richaud De Minzi, 2005; Aluja et al. 2007; Mestre et al. 2007).

\section{Clima sociofamiliar}

En el desarrollo infantil juega un papel fundamental el ambiente presente en el núcleo familiar, sus componentes organizacionales, sociales y físicos impulsan o limitan el desarrollo de los niños y niñas. El ambiente entendido como clima social familiar está constituido por las particularidades psicosociales e institucionales de un grupo familiar y por todo aquello que se origina en su dinámica interna.

El clima social familiar se compone de tres dimensiones (desarrollo, estabilidad y relaciones), dependiendo de cómo se organicen los factores constituyentes de cada dimensión y cómo se manifiesten en un grupo familiar, se enmarcan las familias en un clima social determinado, ya sea cohesivo, disciplinado o sin orientación.

El desarrollo, como componente constitutivo del clima social familiar, enfatiza la función de la familia en términos de despliegue personal. La autonomía hace parte de los factores del desarrollo que estimulan o limitan los alcances personales en los miembros de una familia; esto depende de si padres y madres impulsan o no la seguridad de sus hijos e hijas en sí mismos; la actuación, como segundo factor del desarrollo, dirige las acciones hacia una estructura orientada a la acción competitiva; otros factores que se suman son el intelectualcultural, que significa el grado de interés en las actividades intelectuales y culturales; el social-recreativo, que mide la participación en actividades lúdicas y deportivas; y la moralidadreligiosidad, definida por la importancia dada a las prácticas y los valores éticos y religiosos.

La estabilidad es entendida como la estructura y la formación de la familia y el control que se ejerce entre sus miembros. Está integrada por la organización referida a la relevancia que los padres y las madres le dan a la planificación de las actividades y responsabilidades de la familia, y al control o grado en que la dirección de la vida familiar se ajusta a las reglas y procedimientos establecidos.

Las relaciones se conforman por el grado de comunicación, expresión e interacción presente en la familia, la cohesión entendida como el grado en que padres-madres e hijos se apoyan y ayudan entre sí; la expresividad permitida entre los miembros de la familia; y el conflicto como expresión abierta de la cólera, la agresividad y el desencuentro entre los miembros de la familia (Moos $\&$ Trickett, 1974; Moos, 1981).

Del clima familiar, del grado de intimidad entre padres e hijos, del nivel de implicación en el desarrollo de sus hijos, del apoyo social que proveen los padres y del poder y autoridad presentes depende la influencia que ejercen 
los padres sobre el desarrollo de sus hijos (Molpeceres et al., 1994; Musitu \& Allatt, 1994).

En este estudio se retomaron los estilos de interacción familiar y el clima sociofamiliar como determinantes del desarrollo social de los niños y las niñas, al considerar la importancia de los factores de tipo contextual en la adquisición de repertorios conductuales necesarios para alcanzar un desempeño en habilidades sociales en la infancia. Las interacciones padre-madre-niño/a y las condiciones de vida en términos de afectos, comunicación, libertad, expresión y norma ofrecidas a los niños y las niñas son un factor modelador de aprendizaje. Mediante los estilos de interacción familiar y el clima social familiar los padres, las madres y los adultos significativos propician el desarrollo de capacidades o destrezas sociales específicas necesarias para realizar competentemente una acción interpersonal, una serie de repertorios comportamentales que permiten su adaptación a las exigencias del medio social llamadas habilidades sociales (Elliot \& Gresham, 1991; Caballo, 1993; Hundert, 1995; Monjas, 2000).

Esto permite resaltar el interés de esta investigación, la cual se propuso estudiar aquellos aspectos familiares que predicen el desempeño en habilidades sociales en niños y niñas, de manera más concreta los estilos de interacción familiar y el clima sociofamiliar que pueden propiciar el desarrollo o no de repertorios sociales.
Este estudio resalta la importancia de conocer los grupos familiares de nuestro contexto y sus estilos de interacción familiar y clima social, como punto de partida de estudios posteriores que permitan indagar la predicción presente entre estos y el desempeño social, comunicativo, cognitivo y emocional presente en los niños y las niñas, y desde allí generar planes de acción formativa para los padres y las madres. Este estudio permitió continuar reforzando las ideas existentes frente a la importancia de la familia y la necesidad de crear espacios de interacción con ellas para su formación, posibilitando reforzar a la familia como papel director y coordinador de importantes factores influyentes en el desarrollo del niño y la niña, en la validez de las prácticas de crianza positivas y en la prevención de prácticas negativas que impliquen una génesis o mantenimiento de conductas disfuncionales en los niños y las niñas. La psicología educativa ha centrado su atención en la salud y la infancia, reconociendo que resulta esencial implementar estrategias de prevención que fortalezcan los factores protectores en los diversos contextos, y especialmente en las familias, para tratar de optimizar las influencias ambientales en el desarrollo de los niños y las niñas, contribuir a la generación de factores protectores, al mismo tiempo que prevenir riesgos que puedan alterar el desarrollo de los infantes. Esto es posible en la medida en que se logre 
conocer la familia y las variables que confluyen en ella, como es el caso de los estilos de interacción parental y el clima familiar.

\section{Método}

Esta investigación fue de tipo no experimental, transversal y correlacional, ya que se analizó la influencia del tipo de interacción y clima familiar de los padres y las madres sobre el desarrollo social de sus hijos.

\section{Instrumentos}

Para la evaluación de los estilos de interacción familiar se utilizó la prueba de prácticas educativas familiares (PEF) de García \& Román (2003); esta contiene una escala para adultos, la cual recoge la percepción de padres, madres, tutores o responsables encargados de la educación de los niños y las niñas en el entorno familiar. Las percepciones se recogen a través de tres situaciones: cuando inician algo nuevo, cuando hay una ruptura de rutinas y cuando cuentan o muestran algo. Se obtiene un puntaje total para cada estilo parental: autoritario, equilibrado y permisivo. Cada situación se evalúa mediante 18 ítems para un total de 54. Esta escala ha sido construida y validada por Alonso \& Román (2003) en España, los alfas de Cronbach en cada factor se presentaron de la siguiente manera: estilo autoritario: padres 0.85 ; madres 0.85 ; estilo equilibrado: padres 0.87 , madres 0.89 ; estilo permisivo: padres 0.83 ; madres 0.81. El Alfa de Cronbach de la prueba total es de 0.86 .

Para la evaluación del clima social familiar se utilizó la Escala de clima social familiar (FES) de Moos \& Ticket (1974), formada por 10 subescalas que describen tres dimensiones: relaciones, desarrollo y estabilidad. Las habilidades sociales fueron evaluadas mediante el instrumento creado por Monjas (2000) en el "Programa de enseñanza de HHSS". Ambos instrumentos fueron sometidos a una prueba piloto y a juicios de expertos.

Las habilidades sociales fueron evaluadas mediante el instrumento creado por Monjas (2000) en el "Programa de enseñanza de HHSS". Este instrumento fue sometido a una prueba piloto y a juicios de expertos.

\section{Procedimiento}

- Selección de la muestra: la muestra se estableció por medio de un sorteo a patrones de aleatorización para un total de 108 niños y niñas entre dos y tres años de edad y sus respectivas familias. Esta muestra aleatoria de los niños y las niñas fue seleccionada de una población definida en hogares de bienestar familiar, guardería, iglesias, juntas de acción comunal y bibliotecas lúdicas de los tres niveles socioeconómicos de la ciudad de Medellín (Colombia); este proceso permitió asegurar que los 
resultados en la muestra representarán los resultados en la población dentro de un nivel definido de error muestral.

- Elaboración o prueba de los instrumentos: Se realizó una selección de las pruebas que permitían evaluar las variables del estudio. Se realizó pilotaje y juicio de expertos del Cuestionario de Habilidades Sociales de Monjas (2000) y la Escala de Clima Social Familiar (FES) de Moos \& Ticket (1974), con la finalidad de revisar que el vocabulario original de estas escalas se adecue al contexto de la cultura colombiana.

- Motivación y vinculación de las familias: Se le informó a la comunidad de padres y madres de familia seleccionados en el proceso investigativo, lo concerniente a la metodología, el tipo de pruebas que se utilizarían, el tiempo invertido por ellos y los resultados esperados de la investigación.

- Aplicación de instrumentos: Se realizó en los hogares en compañía de los auxiliares de investigación.

\section{Participantes}

Se seleccionó una muestra de 108 niños y niñas de entre dos y tres años de edad de nivel socioeconómico bajo, medio y alto de la ciudad de Medellín (Colombia) y sus respectivas familias (tabla 1). Este grupo estuvo conforma- do por 54 niñas $(50 \%)$ y 54 niños $(50 \%)$, distribuidos en dos grupos de edad, en los cuales el $62 \%$ de los niños y las niñas tenían 2 años de edad y el $41 \%$ 3 años de edad. Los niños y las niñas pertenecían a los tres niveles socioeconómicos, divididos de la siguiente manera: 35 niños y niñas pertenecen a un nivel socioeconómico bajo $(32,4 \%), 37$ a un nivel medio $(34,3 \%)$ y 36 a un nivel alto (33,3\%). Por último, de los 108 niños y niñas, el $66,7 \%$ se encontraban escolarizados, mientras el otro $33,3 \%$ no estaba cursando ningún nivel educativo (tabla 1).

Tabla 1

Características sociodemográficas de los 108 niños y niñas

\begin{tabular}{lcc}
\hline & Frecuencia & $\%$ \\
\hline Género & & \\
\hline Masculino & 54 & 50 \\
Femenino & 54 & 50 \\
\hline Edad & & \\
\hline 2 años & 67 & 62 \\
3 años & 41 & 38 \\
\hline Nivel socioeconómico & & \\
\hline Bajo & 35 & 32,4 \\
Medio & 37 & 34,3 \\
Alto & 36 & 33,3 \\
\hline Escolaridad & & \\
\hline Sí & 72 & 66,7 \\
No & 36 & 33,3 \\
\hline
\end{tabular}




\section{Resultados}

\section{Modelo explicativo de la influencia de los estilos de interacción familiar y desarrollo de habilidades sociales}

Se realizó un análisis de regresión lineal múltiple teniendo en cuenta las variables que presentaron correlaciones directas o inversas y cuya frecuencia fue importante para este estudio.

La influencia de los estilos de interacción familiar sobre el desempeño en habilidades básicas de interacción social tuvo los siguientes predictores: Trasgresión de la norma del estilo autoritario (se correlaciona negativamente, a mayor utilización de esta estrategia normativa autoritaria por parte de las familias menor desarrollo de repertorios y conductas para relacionarse con las demás personas); y trasgresión de la norma del estilo equilibrado (se correlaciona positivamente, a mayor presencia de un manejo de la norma de manera equilibrada, mayor desarrollo de repertorios para interactuar con los otros), explicando el $14,8 \%$ de la varianza total en la variable Habilidades básicas de interacción social (tabla 2).

La influencia de los estilos de interacción familiar sobre el desempeño en habilidades para hacer amigos y amigas tuvo los siguientes predictores: tipo externo del estilo autoritario (se correlaciona negativamente, a mayores acciones autoritarias de los padres respecto a situaciones externas al niño o la niña, menor desarrollo de conductas apropiadas para relacionarse con los pares); trasgresión de la norma del estilo autoritario (se correlaciona negativamente, a mayor presencia de estrategias autoritarias de los padres como respuesta a la violación de las normas por parte de sus hijos e hijas menor desarrollo de conductas sociales propias para relacionarse con los demás niños y niñas); tipo externo del estilo equilibrado (se correlaciona positivamente, a mayor presencia de acciones equilibradas de los padres sobre problemas de externalización mayor desarrollo de habilidades sociales necesarias para interactuar con los demás niños y niñas); y trasgresión de la norma del estilo equilibrado (se correlaciona positivamente, a mayor presencia de expresiones y acciones equilibradas de los padres como contestación a la violación de las normas por parte de sus hijos e hijas, mayor desarrollo de conductas sociales propias para relacionarse con los demás niños y niñas), explicando el $24,7 \%$ de la varianza total (tabla 2).

Las habilidades conversacionales quedaron con los siguientes predictores: tipo externo del estilo autoritario (se correlaciona negativamente, a mayor utilización de esta estrategia por parte de los padres, menor desarrollo de conductas propicias para enfrentar diversas conversaciones); tipo externo del estilo equilibrado (se correlaciona positivamente, a mayor presencia de este componente en las familias, mayor 
Laura Isaza Valencia \& Gloria Cecilia Henao López

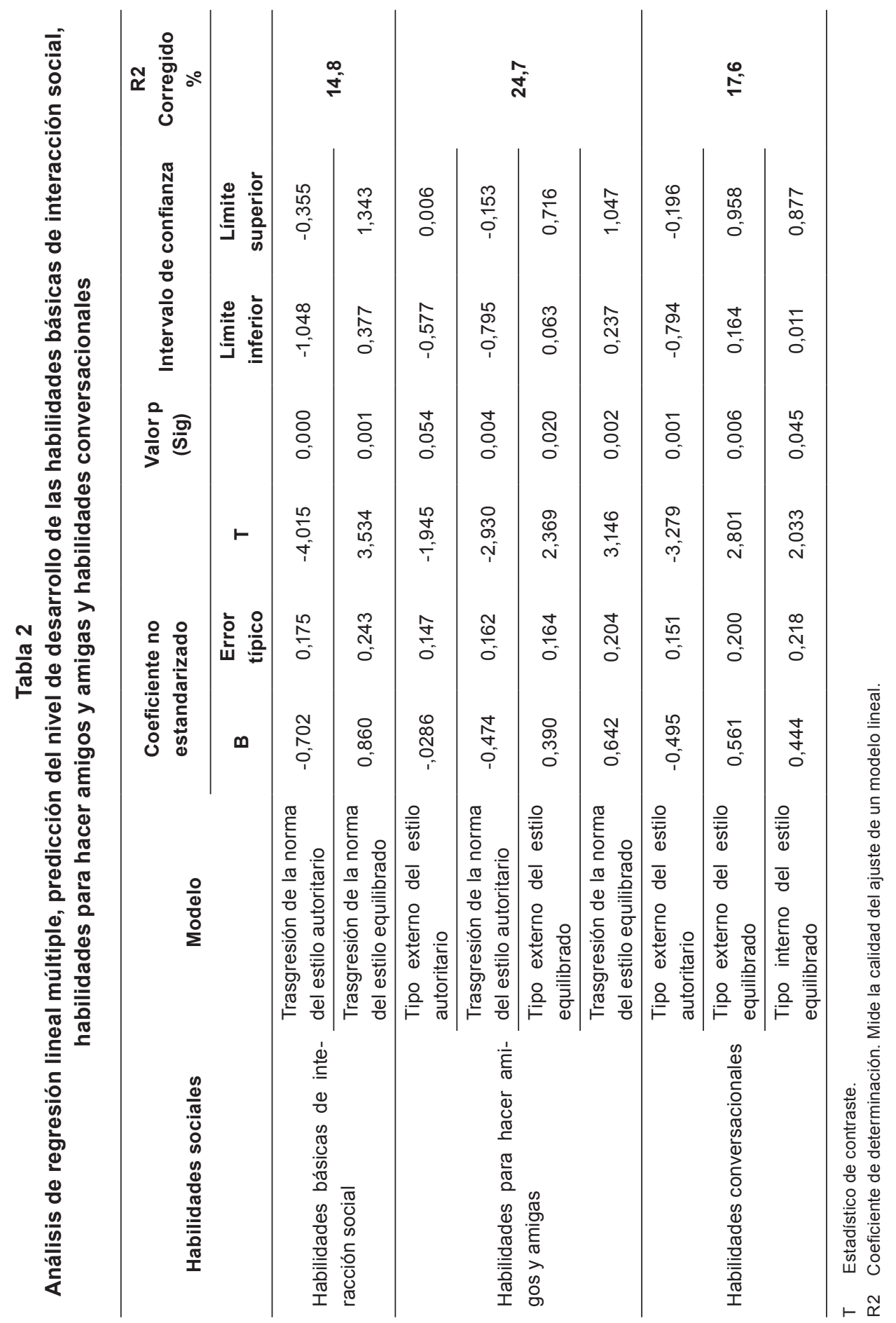


Desarrollo de habilidades sociales en niños y niñas

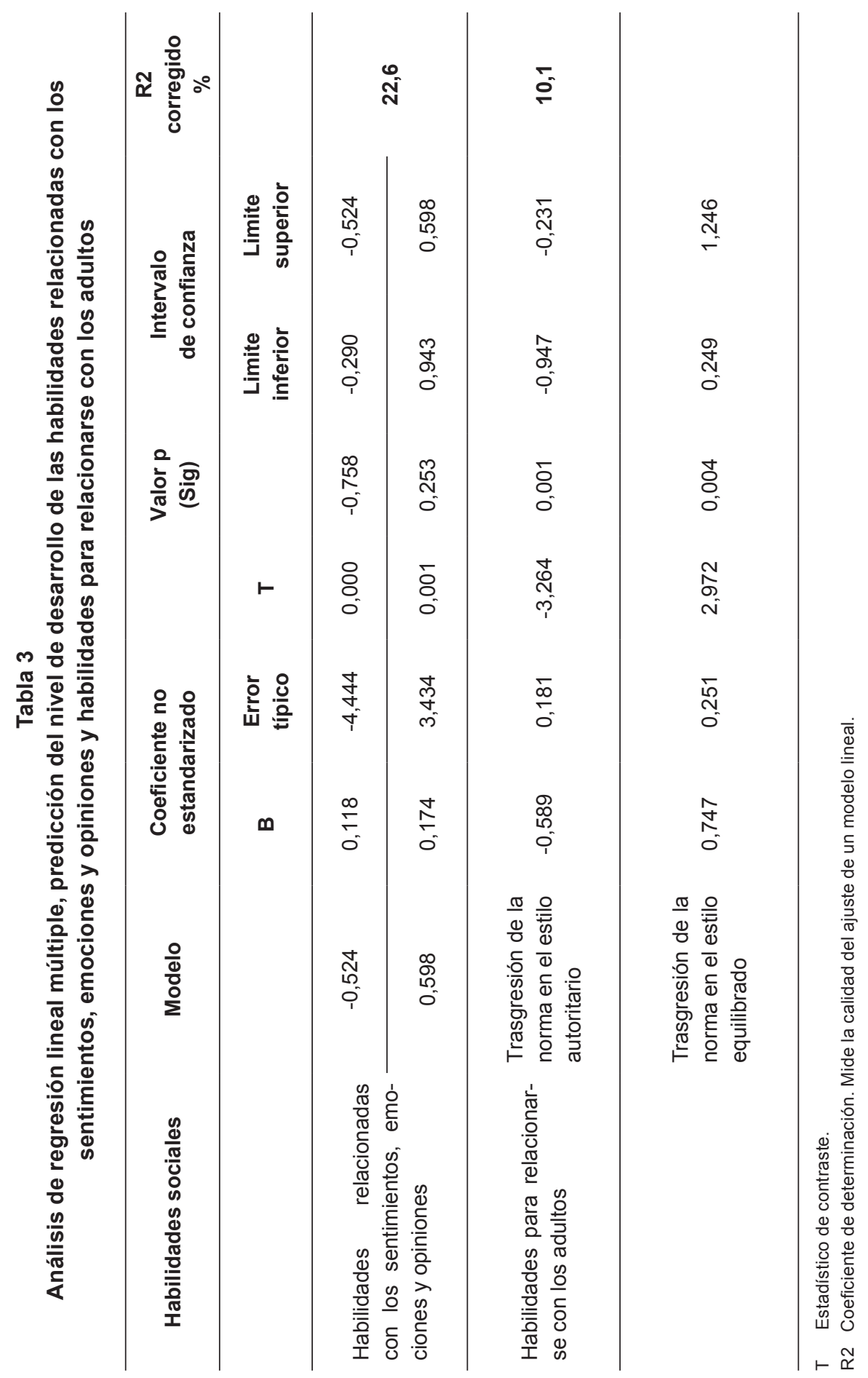


desarrollo de habilidades para participar en conversaciones, y tipo interno del estilo equilibrado (se correlaciona positivamente, a mayor utilización de esta estrategia por parte de los padres, mayor desarrollo de repertorios y conductas conversacionales por parte de los niños y niñas), explicando el 17,6\% de la varianza total en la variable.

La influencia de los estilos de interacción familiar sobre el desempeño en habilidades relacionadas con los sentimientos, emociones y opiniones tuvo los siguientes predictores: tipo externo del estilo autoritario (se correlaciona negativamente, a mayor utilización de esta estrategia por parte de los padres, menor desarrollo de repertorios y conductas para expresarse asertivamente); y tipo interno del estilo equilibrado (se correlaciona positivamente, a mayor utilización de esta estrategia por parte de los padres, mayor desarrollo de habilidades para responder y opinar adecuadamente a nivel emocional); explicando el 22,6\% de la varianza total (tabla 3).

Las habilidades para relacionarse con los adultos presentaron como predictores: trasgresión de la norma en el estilo autoritario (se correlaciona negativamente, a mayor utilización de esta estrategia por parte de los padres, menor desarrollo de repertorios básicos para interactuar con las generaciones posteriores); y trasgresión de la norma en el estilo equilibrado (se correlaciona positivamente, a mayores acciones equilibradas para responder a conductas desafiantes de la norma por parte de los niños y las niñas, mayor presencia de habilidades para interactuar con los adultos), explicando el 10,1\% de la varianza total (tabla 3 ).

\section{Modelo explicativo de la influencia del clima social familiar y desarrollo de habilidades sociales}

Se realizó un análisis de regresión lineal múltiple, teniendo en cuenta las variables que presentaron correlaciones directas o inversas y cuya frecuencia fue importante para este estudio.

La influencia del clima sociofamiliar sobre el desempeño de habilidades básicas de interacción social tuvo los siguientes predictores: cohesión (se correlaciona negativamente, a mayor uso de esta estrategia por parte de las familias menor desarrollo de repertorios y conductas para relacionarse con las demás personas); conflicto en la familia (se correlaciona positivamente, a mayor presencia de este componente del clima sociofamiliar en las familias mayor desarrollo de repertorios para interactuar con los otros), y socio-recreativo (se correlaciona negativamente, a menor utilización de esta estrategia por parte de las familias menor desarrollo de repertorios y conductas para relacionarse con las demás personas), explicando el $15,4 \%$ de la varianza total en la variable habilidades básicas de interacción social (tabla 4). 
Desarrollo de habilidades sociales en niños y niñas

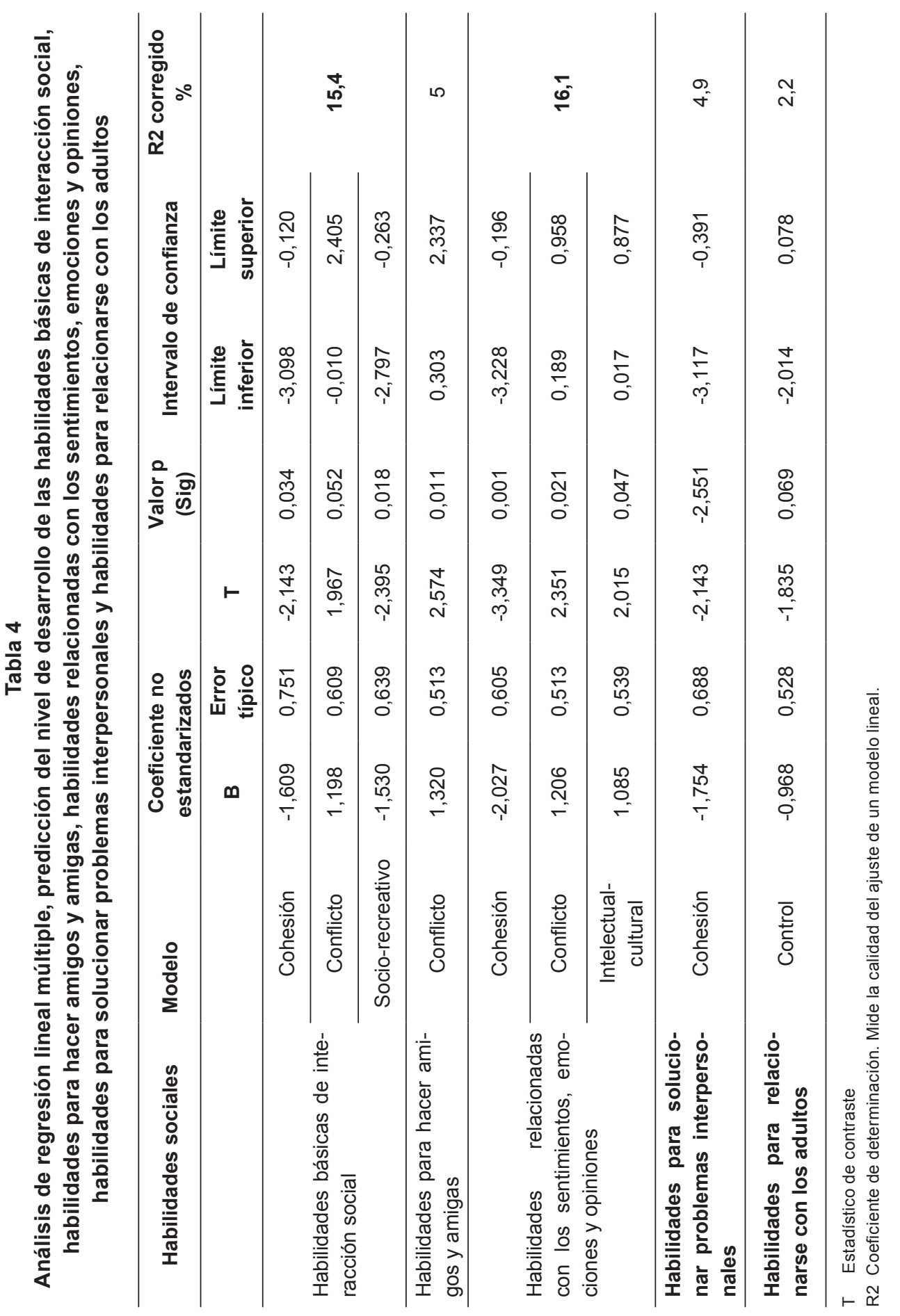


Respecto a la habilidad para hacer amigos y amigas, quedó como predictor el conflicto (se correlaciona positivamente, a mayor presencia de conflicto en las familias mayor desarrollo de conductas apropiadas para relacionarse con los pares), explicando el 5\% de la varianza total.

Las habilidades relacionadas con los sentimientos, emociones y opiniones quedó con los siguientes predictores: cohesión (se correlaciona negativamente, a mayor utilización de esta estrategia por parte de las familias, menor desarrollo de conductas asertivas propicias para enfrentar problemas); conflicto en la familia (se correlaciona positivamente, a mayor presencia de este componente del clima social familiar en las familias, mayor desarrollo de habilidades para expresar emociones y opiniones) e intelectualcultural (se correlaciona positivamente, a mayor utilización de esta estrategia por parte de las familias, mayor desarrollo de repertorios y conductas para enfrentar adversidades sociales), explicando el $16,1 \%$ de la varianza total en la variable habilidades relacionadas con los sentimientos, emociones y opiniones.

Las habilidades para la solución de problemas interpersonales quedó con el predictor cohesión (se correlaciona negativamente, a mayor utilización de esta estrategia por parte de las familias, menor desarrollo de repertorios y conductas para dar respuesta a problemáticas sociales), explicando el $4,9 \%$ de la varianza total.
Las habilidades para relacionarse con los adultos quedó con el predictor control (se correlaciona negativamente, a mayor utilización de esta estrategia por parte de las familias, menor desarrollo repertorios básicos para interactuar con las generaciones posteriores), explicando el $2,2 \%$ de la varianza total.

\section{DisCusión}

En el desarrollo social se da un papel primordial a los componentes socioculturales, en los cuales se reconoce la función socializadora de los padres o cuidadores inmediatos de los niños y las niñas. El tipo relacional manifiesto entre padres e hijos depende en gran medida del estilo de interacción parental estructurado por los padres y del clima social establecido en la organización y dinámica familiar;

“[...] los estilos de interacción familiar y el clima social familiar son variables que en cierta medida generan una explicación y una predicción del desarrollo de habilidades sociales; por esta razón, en los niños y las niñas en quienes se observaba un repertorio básico para iniciar y mantener situaciones sociales, se establece una asociación con el estilo parental equilibrado y con los componentes desarrollo (procesos de desarrollo personal, que son fomentados en el núcleo familiar), relaciones (canales de comunicación y expresión propiciados en la familia) y estabilidad (estructura y organización de la familia y grado de control que los padres ejercen 
sobre sus hijos e hijas) del clima social familiar" (Isaza \& Henao, 2010).

Los padres de familia de este estudio, a través de acciones y verbalizaciones que manifiestan como estrategias para encausar los comportamientos de los hijos y las hijas, presentan características propias de los estilos de interacción parental autoritario, equilibrado y permisivo; estos estilos emergen de acuerdo con los aprendizajes y bagajes socioculturales de las familias y de las situaciones particulares que experimentan en el contacto con los infantes. Cada estilo en relación con las habilidades propicia el desarrollo o no de algunos repertorios sociales, dependiendo de los ambientes sociales, de los espacios de comunicación, de las estructuras de apego y del control de la disciplina; es posible en cierta medida la presencia o no de niveles altos de desempeño social en los niños y las niñas.

Los hallazgos de este estudio respecto a los beneficios del estilo equilibrado a nivel de la dimensión social, apoyan los estudios de Baumrind (1967, 1968, 1970), quien concluyó que los padres equilibrados, que se caracterizan por su constante comunicación y apoyo, así como consistencia y estabilidad normativa, permiten el desarrollo de confianza en sus hijos e hijas; los de García \& Román (2003), quienes consideran el estilo equilibrado como el creador de niveles más altos del desempeño socioemocional de los niños y las niñas; y el estudio de Aluja et al. (2007). Estos investigadores relacionan los valores prosociales y la estabilidad emocional con estilos educativos permeados por el afecto. A modo de conclusión, los resultados de esta investigación permiten considerar que el estilo equilibrado se asocia con el desarrollo de conductas sociales adaptativas.

En contraposición, los padres autoritarios y permisivos, quienes generan un ambiente desestructurado, no propician un despliegue de habilidades sociales, lo cual va en consonancia con los estudios de Valiente et al. (2004), ya que ellos encontraron que los padres con alto nivel de expresión de emociones negativas generaban niños y niñas sin necesidad de ser simpáticos en una situación social. Cuando en los padres predominan expresiones positivas los niños y las niñas enfrentan las situaciones con mejores competencias sociales. Como mencionan McMahon \& Wells (1998), las privaciones y los excesos que muestran los padres en sus repertorios básicos de conducta, cuando desempeñan su papel, constituyen uno de los principales componentes garantes del origen del desarrollo y mantenimiento de los problemas de comportamiento de los niños y las niñas.

Como se observa en este y en otros estudios, los niños y las niñas con padres que presentan un estilo de interacción equilibrado manifiestan un mejor desempeño social, mientras que los niños y las niñas con padres permisivos y autoritarios generan menor repertorio social 
que les posibilite un desempeño social favorable y una competencia social (Isaza \& Henao, 2010; García \& Román, 2005). En resumen, las familias con estrategias equilibradas caracterizadas por el diálogo, las expresiones de afecto y las normas instituidas claramente favorecen en sus hijos e hijas aprendizajes de repertorios sociales más amplios; por el contrario, las familias que se relacionan con sus hijos e hijas desde acciones más punitivas, estrictas y autoritarias, o desde estrategias de poco control y exceso en afecto y permisividad, generan en sus hijos e hijas comportamientos disfuncionales y pobres en aprendizajes adecuados para su edad y contexto (Eisenberg et al., 2001; Stoolmiller, 2001; Chang \& Dodge, 2003).

En el desarrollo de habilidades sociales, la familia presenta un papel relevante; más específicamente, la motivación social que propician los padres desde el clima social estructurado. De esta manera, los padres que generan ambientes sociales novedosos y diversos, de estructura más cohesionada, propician el aprendizaje de repertorios sociales y disminuyen los temores sociales iniciales. Por el contrario, los padres estrictos, inhibidos y retraídos, con estilos más disciplinados y sin orientación, limitan a sus hijos e hijas en su aproximación a situaciones sociales. Así, los niños y las niñas aprenden repertorios de habilidades sociales insuficientes, y respuestas de inhibición o de evitación (Monjas, 2000).
En este estudio se explica la conexión presente entre el clima social familiar con el desarrollo de habilidades sociales; los componentes del clima social: ayuda, apoyo y compenetración de los padres con sus hijos e hijas en niveles moderados; control de la dirección de la vida familiar mediante reglas y procedimientos establecidos; posibilidad de expresar libre y abiertamente la cólera, la agresividad y el conflicto entre los miembros de la familia; participación en actividades recreativas y lúdicas; y presencia de interés en las actividades políticas, sociales, intelectuales y culturales, explican el desempeño favorable de las habilidades sociales en niños y niñas.

En conclusión, el clima sociofamiliar guarda relación con todas las áreas sociales donde priman los componentes presentes en las familias cohesionadas de nuestro estudio, caracterizadas por una estructura y una dinámica democráticas.

\section{RefERENCIAS}

Aguirre, E. (2000). Socialización y prácticas de crianza. En E. Aguirre \& E. Durán (Eds.), Socialización: Prácticas de crianza y cuidado de la salud. Bogotá: CES/Universidad Nacional de Colombia.

Aluja, A., Del Barrio, V., \& García, L. F. (2007). Personalidad, valores sociales y satisfacción de pareja como factores predictores de los estilos 
de crianza parentales. International Journal of Clinical and Health Psychology, 7, 725-737.

Baumrind, D. (1967). Current patterns of parental authority. Developmental Psychology, 28(30), 421-424.

Baumrind, D. (1968). Authoritarian vs. authoritative parental control. Adolescence, 31, 255-271.

Baumrind, D. (1970). Socialization and instrumental competence in young children. Children, 26(2), 104-119.

Baumrind, D. (1971). Current patterns of parental authority. Developmental Psychology Monographs, 4, 1-103.

Bornstein, M. H., Hahn, C.-S., \& Haynes, O. M. (2011). Maternal personality, parenting cognitions, and parenting practices. Developmental Psychology, 47(3), 658-675.

Bronfenbrenner, Urie (1987). La ecología del desarrollo humano. Buenos Aires: Paidós.

Caballo, V. E. (1998). Manual de evaluación y entrenamiento de las habilidades sociales. En M. I. Monjas \& B. González Moreno (Eds.), Las habilidades sociales en el currículo. Madrid: Ministerio de Educación, Cultura y Deporte, Secretaría General de Educación y Formación Profesional, Centro de Investigación y Documentación Educativa.

Chang, S. \& Dodge, M. (2003). Harsh parenting in relation of child emo- tion regulation and aggression. Journal of Family Psychology, 7(4), 598-606.

Eisenberg, N., Losoya, S., Fabes, R. A., Guthrie, I. K., Reiser, M., Murphy, B., Shepard, S. A., Poulin, R., \& Padgett, S. J. (2001). Parental socialization and childrens dysregulated expression of emotion and externalizing problems. Journal of Family Psychology, 15(2), 183-205.

Eisenberg, N., Valiente, C., Morris, A. S., Fabes, R. A., Cumberland, A., Reiser, M., Gershoff, E. T., Shepard, S. A., \& Losoya, S. (2003). Longitudinal relations among parental emotional expressivity, children's regulation, and quality of socioemotional functioning. Developmental Psychology, 39, 2-19.

Elliot, S., \& Gresham, F. (1991). Social skills intervention guide. Austin, TX: Pro-ed.

Fernández, I. (2003). Influencia de los estilos de paternidad en el desarrollo cognoscitivo y socioemocional de los preescolares. Revista de Enseñanza e Investigación en Psicología, 6, 1-29.

García, M. D., Ramírez, G., \& Lima, A. (1998). La construcción de valores en la familia. En M. J. Rodrigo \& Jesús Palacios (Coords.), Familia $y$ desarrollo humano (pp. 201- 221). Madrid: Alianza Editorial. 
García, J., \& Román, J. M. (2003). Escala de identificación de prácticas educativas familiares. Madrid: CEPE.

García, J., \& Román, J. M. (2005). Prácticas educativas familiares y autoestima. Psicothema, 17(1), 76-82.

Grusec, J. E., Goodnow, J. J., \& Kuczynski, L. (2000). New directions in analyses of parenting contributions to children's acquisition of values. Child Development, 71, 205-211.

Hundert, J. (1998). Enhancing social competence in young students. En M. I. Monjas y B. González Moreno (1998). Las habilidades sociales en el currículo, 146. Madrid: Ministerio de Educación, Cultura y Deporte, Secretaría General de Educación y Formación Profesional, Centro de Investigación y Documentación Educativa.

Isaza, L., \& Henao, G. C. (2010). El desempeño en habilidades sociales en niños, de dos y tres años de edad, y su relación con los estilos de interacción parental. Electronic Journal of Research in Educational Psycho$\log y, 8(3)$.

Lansford, J. E., Bornstein, M. H., Dodge, K. A., Skinner, A. T., Putnick, D. L., \& Deater-Deckard, K. (2011). Attributions and attitudes of mothers and fathers in the United States. Parenting: Science and Practice, 11, 199-213.
McMahon, R. J., \& Wells, K. C. (1998). Conduct problems. En E. J. Marsh \& R. A. Barkley (Eds.), Treatment of childhood disorders (2a ed., pp. 111-112). Nueva York: Gilford.

Mestre, M. V., Frías, D., Samper, P., \& Nácher, M. J. (2003). Estilos de crianza y variables personales como factores de riesgo de la conducta agresiva. Revista Mexicana de Psicología, 20(2), 189-199.

Mestre, M. V., Samper, P., Tur, A., \& Díez, I. (2001). Estilos de crianza y desarrollo prosocial de los hijos. Revista de Psicología General y Aplicada, 54, 691-703.

Mestre, M., Tur, A., Samper, P., Nácher, M., \& Cortés, M. (2007). Estilos de crianza en la adolescencia y su relación con el comportamiento prosocial. Revista Latinoamericana de Psicología, 39(2), 211-225.

Molpeceres, M. A., Musitu, G., \& Lila, M. S. (1994). La socialización del sistema de valores en el ámbito familiar. En G. Musitu \& P. Allat (Eds.), Psicosociología de la familia (pp. 121-146). Valencia: Albatros Educación.

Monjas, M. I. (2000). Programa de enseñanza de habilidades de interacción social (PEHIS) para niños y niñas en edad escolar. Madrid: Ciencias de la Educación Preescolar y Especial CEPE. 
Moos, R. H. (1981). Work environment scale. Palo Alto, CA.: Consulting Psychologist Press.

Moos, R. H. \& Trickett, E. J. (1974). Classroom environment scale. Palo Alto, CA.: Consulting Psychologist Press.

Richaud de Minzi, M. C. (2005). Estilos parentales y estrategias de afrontamiento en niños. Revista Latinoamericana de Psicología, 37(1), 47-58.

Rogoff, B. (1993). Aprendices del pensamiento. El desarrollo cognitivo en el contexto social. Barcelona: Paidós.

Solís-Cámara, P., \& Díaz, M. (2007). Relaciones entre creencias y prácticas de crianza de padres con niños pequeños. Anales de Psicología, 23(2), 177-184.
Sroufe, A. (2000). Desarrollo emocional. La organización de la vida emocional en los primeros años. México: Oxford University Press.

Stoolmiller, M. (2001). Synergistic interaction of child manageability problems and parents discipline tactics in predicting future growth in externalizing behavior for boys. Developmental Psychology, 37(6), 814-825.

Valiente, C., Fabes, R. A., Eisenberg, N., \& Spinrad, T. L. (2004). The relations of parental expressivity and support to children's coping with daily stress. Journal of Family Psychology, 18(1), 97-106.

Vigotsky, L. (1979). El desarrollo de los procesos psicológicos superiores. Barcelona: Grijalbo. 